\title{
Lamivudine for Chronic Hepatitis B: A Brief Review
}

\author{
Emilio Palumbo \\ Pediatrics Department of Sondrio Hospital; Italy
}

\begin{abstract}
Until recently, the only generally approved treatment for chronic hepatitis B was alpha-interferon; however, it gives only moderate efficacy in terms of sustained response (biochemical, virological and histological). In fact, only $20 \%$ to $40 \%$ of treated patients respond to therapy, with lower percentages $(\sim 10 \%)$ among patients infected with precoremutant strains of HBV (HBeAb HBV-DNA positive). The FDA of the USA approved the use of lamivudine in adult patients affected by chronic hepatitis $B$ in 1998. In this review, we focused on the pharmacokinetic and pharmacodynamic properties and efficacy and tolerability of lamivudine in the treatment of chronic hepatitis B cases that are both HBeAg and anti-HBe-positive.
\end{abstract}

Key-Words: Lamivudine, hepatitis, HBV.

Despite the use of HBV vaccine, chronic hepatitis B virus (HBV) infection occurs in approximately 5\% of the global population. This infection, if persistent, may lead to chronic hepatitis, cirrhosis and hepatocellular carcinoma in 25 to $40 \%$ of infected patients and is among the 10 main causes of death throughout the world $[1,2]$.

Until recently, the only generally approved treatment for chronic hepatitis B was alpha-interferon; however, it has demonstrated only moderate efficacy in terms of sustained response (biochemical, virological and histological). In fact, only 20 to $40 \%$ of treated patients respond to therapy, with lower percentages ( 10\%) among patients infected with precore-mutant strains of HBV (HBeAb HBV-DNA positive) $[3,4]$. This form, prevalent in the Mediterranean region ( $90 \%$ of all patients with $\mathrm{HBV}$ infection), is due to a mutation at nucleotide 1896 in the precore region of the HBV-DNA genome. The result of this mutation is a stop codon that blocks HBeAg synthesis but still permits HBV replication and hepatitis B core antigen production, leading to persistent viremia and persistent or intermittent elevated serum alanine aminotransferase (ALT) levels, with frequent evolution of the disease into cirrhosis and hepatocellular carcinoma. The suboptimal response of this form to alpha-interferon, with a high rate of non-responders or relapsers, has led to research and development of new antiviral drugs that could be used as alternative therapies.

The use of nucleotide analogues is a milestone in the treatment of chronic hepatitis B (CHB). The FDA of the USA approved the use of lamivudine in adult patients in 1998. This drug is advantageous because of oral administration and good safety parameters; however, it induces a sustained response (after withdrawal of therapy) in only a minority of patients. Thus, the treatment should be given to most patients for long periods. In addition, the long-term efficacy of lamivudine is

Received on 5 May 2008; revised 15 September 2008.

Address for correspondence: Dr. Emilio Palumbo. Department of Pediatric, Hospital of Sondrio. Via Stelvio, 25. Zip code: 23100. Sondrio, Italy. E-mail: emipalu2003@yahoo.it.

The Brazilian Journal of Infectious Diseases 2008;12(5):355-357. (C) 2008 by The Brazilian Journal of Infectious Diseases and Contexto Publishing. All rights reserved. limited by the frequent emergence of drug-resistant HBV mutants [5-7]. Adefovir is a nucleotide analogue associated with a low frequency of resistance; however, its antiviral efficacy is not optimal [8,9].

In this review, we focused on the pharmacokinetics and pharmacodynamics and efficacy and tolerability of lamivudine treatment of chronic hepatitis B cases that are both HBeAg and anti-HBe-positive. Relevant literature was identified through searches of MEDLINE (2002-October 2006). Search terms included, but were not limited to, lamivudine, hepatitis $\mathrm{B}$, pharmacology, pharmacokinetics, adverse events, and therapeutic use.

\section{Pharmacokinetic and Pharmacodynamic Properties}

Lamivudine (3TC), the negative enantiomer of 2'-deoxy-3'thiacytidine, is a dideoxynucleoside analogue used in combination with other agents in the treatment of human immunodeficiency virus type 1 (HIV-1) infection and as monotherapy in the treatment of hepatitis B virus (HBV) infection. It is a nucleoside analog that inhibits HBV-DNA synthesis. Lamivudine undergoes anabolic phosphorylation by intracellular kinases to form lamivudine 5'-triphosphate, the active anabolite that prevents HIV-1 and HBV replication by competitively inhibiting viral reverse transcriptase and terminating proviral DNA chain extension. The pharmacokinetics of lamivudine are similar in patients with HIV-1 or HBV infection, and in healthy volunteers. The drug is rapidly absorbed after oral administration, with maximum serum concentrations usually attained 0.5 to 1.5 hours after administration. The absolute bioavailability is approximately 82 and $68 \%$ in adults and children, respectively. Lamivudine systemic exposure, as measured by the area under the serum drug concentration-time curve (AUC), is not altered when it is administered with food. Lamivudine is widely distributed in total body fluid, the mean apparent volume of distribution (Vd) being approximately $1.3 \mathrm{~L} / \mathrm{Kg}$ following intravenous administration. In pregnant women, lamivudine concentrations in maternal serum, amniotic fluid, umbilical cord and neonatal serum are comparable, indicating that the drug diffuses freely across the placenta. In postpartum women, lamivudine is secreted into breast milk. The concentration of lamivudine in cerebrospinal fluid (CSF) is low to modest, reaching 4\% to $8 \%$ 
of serum concentrations in adults and $9 \%$ to $17 \%$ of serum concentrations in children measured at two to four hours after administration. In patients with normal renal function, about $5 \%$ of the original compound ismetabolized to the transsulphoxide metabolite, which is pharmacologically inactive. In patients with renal impairment, the amount of transsulphoxide metabolite recovered in the urine increases, presumably as a function of decreased lamivudine elimination. As approximately $70 \%$ of an oral dose is eliminated in the urine as unchanged drug, the dose needs to be reduced in patients with renal insufficiency. Hepatic impairment does not affect the pharmacokinetics of lamivudine. Systemic clearance following single intravenous doses averages 20 to $25 \mathrm{~L} / \mathrm{h}$ (approximately $0.3 \mathrm{~L} / \mathrm{h} / \mathrm{Kg}$ ). The elimination half-life of lamivudine is approximately five to seven hours, and the in vitro intracellular half-life of its active 5'-triphosphate anabolite is 10.5 to 15.5 hours and 17 to 19 hours in HIV-1 and HBV cell lines, respectively.

\section{Efficacy and Tolerance}

Randomized controlled trials have demonstrated the efficacy of lamivudine in the treatment of HBeAg-positive and HBeAg-negative CHB. One randomized, placebocontrolled trial showed that almost all patients treated with lamivudine (98\%) had a reduction of serum HBV-DNA levels. Serum HBV-DNA levels were undetectable after lamivudine therapy in $44 \%$ of patients, compared with $16 \%$ of patients treated with a placebo. After one year of treatment, the HBeAg seroconversion rates were 17 and $6 \%$ in the lamivudine and placebo groups, respectively. ALT levels were normalized in $41 \%$ of the lamivudine-treated patients compared with $7 \%$ of the placebo group. Histological improvement was observed in $52 \%$ of the lamivudine group compared with $23 \%$ of the placebo group [5]. The rates of virologic, biochemical and histological response in another randomized controlled trial were similar, with an HBeAg anti-HBeAb seroconversion rate of $21 \%$ [6]. The tolerability and safety of lamivudine were excellent, and the frequency of adverse events was similar to that of the placebo group. Lamivudine therapy seems to be well-tolerated for up to five years; however, data on long-term therapy with lamivudine are limited to a small number of patients. The main limitation of lamivudine treatment is the high rate of viral resistance due to mutations in the YMDD motif of the HBV polymerase gene. Indeed, even if the HBeAg seroconversion rate is increased by continuing treatment, the frequency of resistance to lamivudine increases with time: $24 \%$ at one year, $38 \%$ at two years, $50 \%$ at three years, and $67 \%$ at four years [7]. The most important mutation is substitution of valine or isoleucine for methionine. In many patients, this mutation isaccompanied by a second mutation, in which methionine is substituted for leucine in an upstream region (rtL180M). Lamivudine resistance is more likely to occur in patients with high baseline levels of serum HBV-DNA. Emergence of lamivudine resistant mutants is usually associated with a breakthrough of hepatitis, resulting in moderately increased levels of serum HBV-DNA and ALT, although these levels may remain lower than baseline (pretreatment) for several months.

In another investigation, $76 \mathrm{HBeAg-negative} \mathrm{patients} \mathrm{with}$ CHB were given $100 \mathrm{mg}$ LAM daily in a five-year follow-up study [10]. The incidence of YMDD was $39 \%$ at month 12 , $54 \%$ at month 24 and $57 \%$ at month 36; at the same time, the response declined from $67 \%$ at six months to $51 \%$, $34 \%$ and 29\% after 12, 24 and 36 months, respectively. A complete analysis at month 24 of follow-up was available. Increases in HBV-DNA and ALT levels were apparent in patients with YMDD variants. Among the patients who did not develop YMDD variants, 72\% had normal ALT and negative HBVDNA, whereas only $5 \%$ of the patients who developed YMDD mutants maintained normal ALT levels after one year. In a recent study the efficacy of LAM administered for three years in patients with chronic active anti-HBe-positive hepatitis was evaluated. Thirty-four patients with chronic active anti-HBe-positive hepatitis were treated with LAM (100 mg) once daily for three years. Before treatment, all patients had serum ALT levels $>$ two times normal levels for $>$ six months and HBV DNA positivity $>5 \mathrm{pg} / \mathrm{mL}$, as determined by a sandwich hybridization test for nucleic acids. Both ALT and HBV DNA were monitored during therapy. After 12 months of therapy, 24 of 34 patients (70.6\%) showed evidence of HBV DNA clearance and normal ALT levels; 22 of 34 (64.7\%) and 19 of 34 (55.8\%) patients maintained a complete response after two and three years of therapy, respectively. Long-term LAM therapy (>one year) was not associated with an increase in the response of initially nonresponding patients. The YMDD variant emerged in $17.6 \%$ of patients in the first year, in $35.2 \%$ during the second year, and in 52.9\% during the third year of treatment. LAM was well tolerated during the three-year therapy in all patients. In patients with chronic active anti-HBe-positive hepatitis, the LAM response rate tended to decrease over time due to the emergence of YMDD variants [11].

\section{Conclusions}

The goal of therapy for patients with HBV infection is to prevent the progression of liver disease to cirrhosis and hepatic cell cancer. In recent years, progress has been made in the treatment of CHB. Nucleos(t)ide analogs such as lamivudine have been approved as initial therapy for CHB. Currently, lamivudine is the first line of treatment of chronic hepatitis B; however, efficacy is limited by the high frequency of resistance. Recent preliminary results show that adefovir dipivoxil and entecavir could be potent analogs for the treatment of HBV. Further studies are being made to assess the long-term efficacy and safety of these drugs [12,13].

\section{References}

1. Chu C.M., Liau Y.F. Natural history of chronic B virus infection: an immunopathological study. J Gastroenterol Hepatol 1997;12, S218-S22. 
2. Ganem D., Prince A.M. Hepatitis B virus infection-natural history and clinical consequences. N Engl J Med 2004;350:1118-29.

3. Olivero F., Santantonio T., Bellati G., et al. Long-term response to therapy of chronic anti-Hbe-positive hepatitis B is poor independent of type and schedule of interferon. Am J Gastroenterol 1999;94:1366-72.

4. van Zonneveld M., Honkoop P., Hansen B.E., et al. Long-term follow-up of alpha-interferon treatment of patients with chronic hepatitis B. Hepatology 2004;39:804-10.

5. Lai C.L., Chien R.N., Leung N.W., et al. A one year trial of lamivudine for chronic hepatitis B. N Engl J Med 1998;339:61-8.

6. Dienstag J.L., Schiff E.R., Wright T.L., et al. Lamivudine as initial treatment for chronic hepatitis B in the United States. N Engl J Med 1999;341:1256-63.

7. Lau D.T., Khokhar F., Doo E., et al. Long-term therapy of chronic hepatitis B with lamivudine. Hepatology 2000;32:828-34.
8. Zeng M., Mao Y., Yao G., et al. A double-blind randomized trial of adefovir dipivoxil in Chinese subjects with HBeAg. positive chronic hepatitis B. Hepatology 2006;44(1):108-16.

9. Hadziyannis S.J., Tassopoulos N., Heathcote E.J., et al. Adefovir dipivoxil for the treatment of hepatitis $\mathrm{B}$ e antigen-negative chronic hepatitis B. N Engl J Med 2003;348:800-7.

10. Rizzetto M., Tassopoulos N.C., Goldin R.D., et al. Extended lamivudine treatment in patients with HBeAg-negative chronic hepatitis B. J Hepatol 2005;42(2):158-62.

11. Scotto G., Palumbo E., Fazio V., et al. Prolonged lamivudine treatment in patients with chronic active anti-HBe positive hepatitis. Am J Ther 2006;13(3):218-22.

12. Hadziyannis S.J. New developments in the treatment of chronic hepatitis B. Expert Opin Biol Ther 2006;6(9):913-21.

13. Zhou X.X., Littler E. Nucleoside analogs as anti-HBV agents. Curr Top Med Chem 2006;6(9):851-65. 